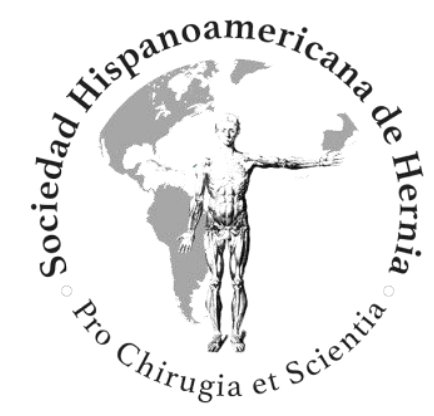

Reflexiones personales sobre la cirugía actual de las hernias

\title{
Personal reflections on current hernia surgery
}




\section{Reflexiones personales sobre la cirugía actual de las hernias Personal reflections on current hernia surgery}

El tratamiento quirúrgico de las hernias de la pared abdominal ha sido siempre un apartado frecuente dentro de la cirugía general, si bien no se ha considerado como capítulo de importancia hasta la mitad del siglo Xx, cuando la aparición determinadas circunstancias (que luego analizaremos) modificaron su valoración. Actualmente este capítulo de la cirugía se ha convertido en paradigmático en múltiples servicios de cirugía de todos los continentes.

Las técnicas quirúrgicas tradicionales adolecían de problemas importantes, como la alta tasa de recidivas o la presencia de dolor crónico posoperatorio, que condicionaban su funcionalidad y la consideración de cirugía importante, a pesar de ser uno de los apartados mas frecuentes dentro de cualquier servicio de cirugía general.

La descripción de nuevas técnicas quirúrgicas, la utilización de biomateriales y su aceptación por nuestro organismo sin la presencia de rechazo, la disminución llamativa de las recidivas y de otras complicaciones, los buenos resultados a largo plazo de estos nuevos procedimientos, la cirugía ambulatoria y los avances de las técnicas anestésicas han facilitado y han favorecido el auge de la cirugía herniaria hasta el punto de que hoy en día todos los congresos quirúrgicos tienen un capítulo muy significativo que se ocupa de la cirugía de las hernias de la pared abdominal.

La situación actual en estos inicios del siglo XXI ha sido posible debido a los sucesivos acontecimientos que han tenido lugar en la segunda mitad del siglo xx. En primer lugar hay que citar a Usher, quien, en 1958, desarrolló la malla de polipropileno y su utilidad en la cirugía herniaria.

Otro punto determinante fue la descripción por Liechtenstein de su técnica quirúrgica con la introducción del término tensión free, que dio lugar a una modificación de los conceptos que hasta ese 
momento se tenían sobre la reparación herniaria y que ha condicionado la descripción de numerosos procedimientos basados en esta idea.

Esta revolución en el concepto del tratamiento de las hernias fue el punto de partida de otras innovaciones que han ido introduciéndose para lograr unos mejores resultados: nuevos materiales, mallas de bajo peso molecular, pegamentos, mallas reabsorbibles, abordajes laparoscópicos, etc., que han propiciado unos resultados excelentes tanto en el seguimiento a largo plazo como en la disminución llamativa de la tasa de recidivas, la disminución de las complicaciones usuales, como el dolor crónico, y la reincorporación temprana a la actividad social y personal de los pacientes.

¿Qué circunstancias han tenido lugar en el devenir del siglo Xx para que la cirugía herniaria, considerada como cirugía de escasa relevancia durante todo el desarrollo de la cirugía moderna, se haya convertido, hoy en día, en un proceso de gran relevancia quirúrgica? Los cirujanos que han desarrollado los conceptos actuales de la cirugía se han preocupado y han estudiado durante muchas décadas los problemas médicos relacionados con los órganos importantes del aparato digestivo (el esófago, el estómago, el hígado, las vías biliares, el páncreas, el colon, etc.) y han encaminado sus esfuerzos al desarrollo de técnicas quirúrgicas para las patologías de estos órganos, pero no se han preocupado de los aspectos relacionados con la pared abdominal (hernias, eventraciones, etc.).

No obstante, durante muchos lustros, pequeños grupos de cirujanos y de anatomistas de diferentes países han mantenido viva la llama de la cirugía de la pared abdominal. Los más significados fueron el grupo francés (Groupe Recherche Européen sur la Paroi Abdominale, GREPA), en el que hay que citar a Stoppa, Alexandre y Chevrel, entre otros, y el grupo americano formado por cirujanos de renombre como Nyhus, Wantz, Gilbert, Bendavid, etc. Tanto uno como otro celebraban pequeñas reuniones periódicas para contrastar opiniones, resultados y complicaciones, y sus publicaciones científicas en revistas de 
reconocido prestigio hicieron que los grupos fueran ampliándose con cirujanos de otros países: de Italia, España, Bélgica, Alemania, etc., en el europeo, y de México, Canadá, Brasil o Argentina en el grupo americano. Todo ello fue la base para la creación de la Sociedad Europea de la Hernia (EHS) y la americana (AHS), que fue el inicio de la universalización de este entramado quirúrgico y que posteriormente dio lugar a la creación de otras asociaciones, como la Sociedad Hispanoamericana de Hernia, y a capítulos y a secciones nacionales, de las que una de las primeras fue la sección española de pared, creada oficialmente en 1996, aunque su actividad había comenzado como grupo reducido en 1991.

Otro hito fundamental que ha contribuido al desarrollo de esta patología es la existencia de las revistas médicas monográficas (Hernia, en ingles, y Revista Hispanoamericana de Hernia, en español), que han posibilitado la publicación de cientos de estudios y de trabajos específicos sobre la cirugía de la pared abdominal.

\section{¿Cuál fue el punto de inflexión para la eclosión y la} generalización de esta cirugía? Durante unas décadas, los grupos americanos y europeos celebraron reuniones y congresos anuales y reducidos con pequeños grupos de participantes (en Francia y en Estados Unidos). Posteriormente, estos eventos se celebraron en otros países. En 1997, la reunión del GREPA se celebró en Nápoles, a la que asistimos varios miembros de la sección española, donde solicitamos y se nos concedió que la sede para el congreso de 1999 tuviera lugar en Madrid.

Los miembros de la sección decidimos darle un carácter más universal, para lo que fue necesario realizar una amplia difusión del congreso en múltiples países de los distintos continentes, hecho que se vio favorecido por el cambio de denominación de GREPA a European Hernia Society y que dio lugar a que el primer congreso, con la denominación de Congreso Europeo de la EHS, tuviera lugar en Madrid. Asimismo, creímos que era necesario dar voz al mayor 
número posible de participantes. El esfuerzo mereció la pena, pues el número de cirujanos que se congregó superó los 1500 y se presentaron más de 1100 comunicaciones en sus distintos formatos (orales, vídeos y pósteres).

El impacto que produjo el congreso fue muy superior al pensado inicialmente, pues nunca se habían reunido tal número de participantes para un tema monográfico como la cirugía de la hernia. Tanto fue así que los responsables de las direcciones de la EHS y de la AHS nos convocaron durante las sesiones del evento a reuniones específicas para conocer qué mecanismos y acciones habíamos realizado para lograr el importante número de congresistas, cuando en reuniones anteriores la cifra de participantes siempre fue inferior a 400.

En nuestra respuesta indicamos que fueron varias las circunstancias que influyeron para tal cambio: en primer lugar, dar participación activa al mayor número de congresistas en forma de comunicaciones o ponencias; en segundo lugar, la existencia de traducción simultánea en la presentaciones (español-inglés) y, como último apartado, conseguir involucrar a las empresas implicadas en esta cirugía para que su contribución fuese importante, ya que a largo plazo implicaría más beneficios para sus productos.

Los responsables europeos y americanos tuvieron muy en cuenta nuestras consideraciones, lo que se tradujo en la realización de congresos conjuntos de la EHS y de la AHS, con una participación mucho mayor que en los realizados con anterioridad y a los que posteriormente se ha sumado la Sociedad Hispanoamericana de la Hernia y la Asociación Asia-Pacífico de la Hernia.

\section{¿Qué logros se han conseguido con esta universalización de la cirugía de la hernia?}

La difusión y la normalización en todos los continentes de las técnicas quirúrgicas actuales, la aparición de guías clínicas de actuación ante problemas concretos de esta patología, la consolidación de las 
revistas monográficas (Hernia y Revista Hispanoamericana de Hernia ), con aumento significativo de sus factores de impacto debido a la calidad de sus artículos, el interés de múltiples cirujanos de todos los continentes por esta patología y ha propiciado que un considerable número de ellos sea referencia en todo el mundo en esta patología por sus trabajos y sus publicaciones.

Es preciso hacer constar asimismo que la divulgación y la universalización de esta cirugía, con sus propuestas de nuevas técnicas quirúrgicas, el uso de biomateriales, etc., han contribuido de una manera importante a una disminución importantísima de las recidivas herniarias y de otras complicaciones que siempre estaban presentes en la cirugía convencional o tradicional. Hay que destacar también la consolidación de la cirugía mayor ambulatoria en esta patología y la generalización de los métodos anestésicos que posibilitan las propuestas anteriores y que facilitan el alta precoz de los pacientes y la reincorporación temprana a su actividad habitual.

La conclusión de todos los apartados anteriores sería que la cirugía de la pared abdominal ha logrado en las últimas décadas una gran excelencia, pues sus técnicas son fácilmente reproducibles y su uso se ha generalizado en todos los países, los resultados son excelentes, las complicaciones casi han desaparecido y el coste económico de esta cirugía es muy bajo. Por lo tanto, habría que pensar que la medicina ha conseguido solucionar de manera definitiva uno de los problemas más comunes y frecuentes de la patología quirúrgica, pero, en honor a la verdad, hay nuevas circunstancias que ponen en cuestión estas conclusiones, y me refiero al uso de la cirugía robótica en la pared abdominal.

En determinados servicios quirúrgicos ha comenzado a realizarse la cirugía herniaria con este procedimiento y ya existen publicaciones referidas a ella. Mi reflexión me lleva a las siguientes cuestiones sobre el uso de la robótica: ¿ha mejorado los resultados de otras técnicas? ¿Ha disminuido la estancia hospitalaria? ¿Es una técnica fácilmente reproductible? ¿Es posible su utilización en todos los países? 
¿Disminuye el coste económico? Mi impresión personal es que todas las preguntas se responden negativamente y creo que el uso de la cirugía robótica en la cirugía de pared abdominal está en relación más con el ego personal de algunos cirujanos que con el beneficio para los pacientes, y únicamente en algún caso específico podría tener una indicación clara.

La actualidad nos dice que la cirugía actual de los defectos de la pared abdominal cumple todos los criterios que se requieren para una cirugía de excelencia y que en el futuro se beneficiará de mejoras puntuales, pero siempre bajo los criterios anteriores de buenos resultados, mínimas complicaciones y bajo coste económico.

Manuel Hidalgo Pascual

Universidad Nacional Autónoma de México. Ciudad de México (México)

Correo electrónico: manuel.hidalgo@salud.madrid.org

\section{BIBLIOGRAFÍA RECOMENDADA}

1. Donkor C, González A, Gallas MR, Helbig M, Weinstein C, Rodríguez J. Current perspectives in robotic hernia repair. Robot Surg. 2017;4:57-67. DOI: 10.2147/RSRR.S101809. eCollection 2017

2. Lichtenstein I, Shulman AG, Amid PK, Montllor MM. The tensionfree hernioplasty. Am J. Surg. 1989;157(2):188-93.

3. Mckernan JB, Laws H. Laparoscopic preperitoneal prosthetic repair of inguinal hernias. Surg. Rounds 1992;15:597-608.

4. Usher FC, Oschener J, Tuttle LL. Use of marlex mesh in the repair of incisional hernias. Am. Surg. 1958;24:969-72. 